\title{
Ergoregion instability of a rotating quantum system
}

\author{
Leandro A. Oliveira, ${ }^{1,2, *}$ Luis J. Garay, ${ }^{3, \dagger}$ and Luís C. B. Crispino ${ }^{2, *}$ \\ ${ }^{1}$ Campus Salinópolis, Universidade Federal do Pará, 68721-000 Salinópolis, Pará, Brazil \\ ${ }^{2}$ Faculdade de Física, Universidade Federal do Pará, 66075-110 Belém, Pará, Brazil \\ ${ }^{3}$ Departamento de Física Teórica, Universidad Complutense de Madrid, 28040 Madrid, Spain \\ and Instituto de Estructura de la Materia (IEM-CSIC), Serrano 121, 28006 Madrid, Spain
}

(Received 7 March 2018; published 26 June 2018)

\begin{abstract}
Using the analogy between acoustic perturbations in an ideal fluid and the description of a Klein-Gordon scalar field in a curved spacetime, we study the quasinormal modes of a quantum system: the rotating BoseEinstein condensate. To compute quasinormal frequencies, we use two different numerical techniques, namely the direct integration and the continued-fraction methods. We study in detail the ergoregion instability of this linearly perturbed system, comparing the results with different setup configurations.
\end{abstract}

DOI: 10.1103/PhysRevD.97.124063

\section{INTRODUCTION}

Acoustic analogue systems have shown to be interesting alternatives to investigate (theoretically and experimentally) some properties of compact objects [1-5], as black holes, which so far cannot be subject to experimental reproduction in laboratory. Among the properties that can be studied in acoustic analogue systems, stand out: absorption and scattering of waves [6-9], quasinormal modes [10-13], ergoregion instability [14-17], and stationary configurations [18,19]. Acoustic analogues have been studied in various systems, among which we can mention the perfect fluids and BoseEinstein condensates (BECs) [20-22], the latter being shown as a fruitful proposal of an experimental setup. Recently an experimental apparatus in BECs was used for attempts to observe some aspects of Hawking radiation in acoustic analogue systems [23,24].

In this work we investigate the instability of a rotating BEC. Previous studies on the instability of acoustic analogue systems were performed for incompressible [14] and for compressible [15-17] (perfect) fluids. As essential tools for the investigation of instabilities, there are the quasinormal modes (QNMs) of a system. QNMs are associated with purely outgoing modes at spatial infinity, each mode being described by a complex frequency [25-27]. As a purely circulating system, we describe the effective spacetime of a compressible hydrodynamic vortex [15,28], compatible with an experimental setup in a rotating BEC. QNMs were previously studied in one-dimensional flows in a BEC with steplike discontinuity [29]. Furthermore, the scattering of the sound waves was studied for a hydrodynamic vortex with a density profile of a BEC [30].

\footnotetext{
*laoliveira@ufpa.br

tuisj.garay@ucm.es

*crispino@ufpa.br
}

The remainder of this paper is structured as follows. In Sec. II we describe acoustic spacetimes in the context of a rotating compressible fluid. In Sec. III we describe a purely circulating BEC as a rotating acoustic analogue. In Sec. IV we study the propagation of linear perturbations in this compressible system, using the description in the frequency domain. In Sec. V we obtain the QNM frequencies of this system using two different methods: direct integration (DI) and continued-fraction (CF). In Sec. VI we investigate the ergoregion instability of the BEC hydrodynamic vortex, validating and commenting our results, comparing the QNM frequencies obtained via DI and CF methods. We conclude with a brief discussion in Sec. VII.

\section{EFFECTIVE SPACETIMES OF ROTATING ACOUSTIC ANALOGUES}

Requiring a fluid flow to be irrotational (i.e., with zero vorticity), namely

$$
\nabla \times \vec{v}=0
$$

where $\vec{v}$ is the flow velocity, we may write

$$
\vec{v}=-\nabla \Phi,
$$

where $\Phi$ is the velocity potential.

We may describe the irrotational flow of an inviscid fluid without thermal conductivity (ideal fluid) using the Euler equation, as follows:

$$
\frac{\partial \vec{v}}{\partial t}+\frac{1}{2} \nabla|\vec{v}|^{2}+\frac{\nabla P}{\rho}+\nabla V_{\mathrm{ext}}=0
$$

where $V_{\text {ext }}$ is an external potential, $P$ is the pressure and $\rho$ is the mass density of the fluid. We consider that the ideal fluid is barotropic, i.e., with an equation of state $(\mathrm{EoS})$ such that 


$$
P=P(\rho)
$$

Furthermore, we impose mass conservation, which may be described by the continuity equation, namely

$$
\frac{\partial \rho}{\partial t}+\nabla \cdot(\rho \vec{v})=0 .
$$

Using the description for effective curved spacetimes in ideal fluids proposed by Unruh [1], we may obtain from Eqs. (2)-(5) that the propagation of linear disturbances in the background flow can be governed by the Klein-Gordon equation [2], namely

$$
\nabla_{\mu} \nabla^{\mu} \phi=\frac{1}{\sqrt{|g|}} \partial_{\mu}\left(\sqrt{|g|} g^{\mu \nu} \partial_{\nu} \phi\right)=0,
$$

with $g^{\mu \nu}$ being the contravariant effective metric, $g \equiv \operatorname{det}\left(g_{\mu \nu}\right)$, and $\phi$ is the velocity potential associated to the linear perturbations, i.e.,

$$
\delta \vec{v}=-\nabla \phi,
$$

where $c_{\mathrm{S}}$ is the speed of sound, which can be written as

$$
c_{\mathrm{s}} \equiv \sqrt{d P / d \rho} .
$$

An appropriate way to describe the properties of the effective spacetime of a rotating acoustic analogue is adopting a cylindrical coordinate system $(t, r, \theta, z)$. Here we focus on the hydrodynamic vortex as a rotating acoustic analogue - a purely circulating fluid — whose flow velocity $\vec{v}=v_{r} \hat{r}+v_{\theta} \hat{\theta}+v_{z} \hat{z}$ is such that both the radial $v_{r}$ and the z-component $v_{z}$ vanish, so that

$$
\vec{v}=v_{\theta} \hat{\theta} .
$$

Furthermore, from Eq. (1), we find that the velocity flow may be written as

$$
\vec{v}=\frac{C}{r} \hat{\theta},
$$

where $C$ is a constant related with the conserved circulation.

From the Klein-Gordon equation, given by Eq. (6), we may write, in cylindrical coordinates, the contravariant effective metric $g^{\mu \nu}$ for a hydrodynamic vortex, namely

$g^{\mu \nu}=-\frac{1}{\rho c_{\mathrm{S}}}\left[\begin{array}{cccc}1 & 0 & v_{\theta} / r & 0 \\ 0 & -c_{\mathrm{S}}^{2} & 0 & 0 \\ v_{\theta} / r & 0 & \left(v_{\theta}^{2}-c_{\mathrm{S}}^{2}\right) / r^{2} & 0 \\ 0 & 0 & 0 & -c_{\mathrm{S}}^{2}\end{array}\right]$.

Thus, the line element $d s^{2}=g_{\mu \nu} d x^{\mu} d x^{\nu}$ of a hydrodynamic vortex can be written as [31-34] $d s^{2}=\frac{\rho}{c_{\mathrm{s}}}\left[-c_{\mathrm{s}}^{2} d t^{2}+\left(r d \theta-\frac{C}{r} d t\right)^{2}+d r^{2}+d z^{2}\right]$.

Note that the line element (12) may be written as a function of a quantity only, namely the fluid density $\rho$, since the speed of sound explicitly depends on the density [as may be seen from Eq. (8)]. Thus, to study the properties of the hydrodynamic vortex it is necessary to know the fluid density profile. Here we consider that the density $\rho$ and the speed of sound $c_{\mathrm{s}}$ are functions of the radial coordinate only, obtained from local properties of the unperturbed fluid flow [2]. Furthermore, as can be seen from the line element (12), the spacetime of the hydrodynamic vortex has no event horizon, but has an ergoregion delimited by an outer boundary $r_{\mathrm{e}}$, which can be obtained from $[2,14,15]$

$$
c_{\mathrm{s}}\left(r=r_{\mathrm{e}}\right)^{2}=\left|\vec{v}\left(r=r_{\mathrm{e}}\right)\right|^{2} .
$$

Next, we will obtain the expressions for the density $\rho$ and the speed of sound $c_{\mathrm{s}}$, as functions of $r$, for the hydrodynamic vortex with a compatible experimental setup in a BEC.

\section{BEC AS A ROTATING ACOUSTIC ANALOGUE}

The BEC considered in this work is described as a physical system obtained when a set of bosonic particles, which are subjected to an exterior potential and interact in pairs with neighbor particles, occupy the same quantum state-the ground quantum state of the system [20-22,35,36]. This system is formed at the limit in which the temperature of the bosons is decreased to extremely low values $(T \approx 0)$. At zero temperature, all particles of the condensate occupy the quantum ground state. This system can be described by a wave function, whose evolution is governed by the GrossPitaevskii equation [35,36], namely

$$
i \hbar \frac{\partial \Psi}{\partial t}=\left(-\frac{\hbar^{2}}{2 M} \nabla^{2}+V_{\text {ext }}(\vec{r})+U|\Psi|^{2}\right) \Psi,
$$

where $\hbar$ is the reduced Planck constant, $V_{\text {ext }}(\vec{r})$ is an external potential, $\Psi$ is a quantum field that describes the BEC, $M$ is the individual mass of each boson and $U$ parametrizes the strength of the interaction between bosons [35,36], as

$$
U=\frac{4 \pi a \hbar^{2}}{M},
$$

with $a$ being the scattering length between two bosons in the condensate $[35,36]$.

The total number of bosons in the condensate is given by $N=\int d x^{3}|\Psi|^{2}$ and the density of bosons of the BEC can be written as

$$
\rho=|\Psi|^{2} .
$$

We may rewrite the Gross-Pitaevskii equation using the Madelung representation for the wave function $\Psi$, namely 


$$
\Psi=\sqrt{\rho} \exp \left[-\frac{i}{\hbar}\left(\Phi(t, \vec{r})+\mu_{\mathrm{c}} t\right)\right]
$$

where $\rho$ is the density of the Madelung fluid [22] and $\mu_{\mathrm{c}}$ is the chemical potential (here we consider $\mu_{\mathrm{c}}$ fixed $[35,36]$ ).

Substituting Eq. (17) in the Gross-Pitaesvskii equation (14), we obtain the following expression:

$$
\begin{aligned}
& -\frac{i \hbar \rho^{-\frac{1}{2}}}{2} \frac{\partial \rho}{\partial t}-\sqrt{\rho} \frac{\partial \Phi}{\partial t}-\frac{\hbar^{2}}{2 M} \nabla^{2} \sqrt{\rho}+\frac{i \hbar}{M}(\nabla \sqrt{\rho}) \cdot(\nabla \Phi) \\
& +\frac{\sqrt{\rho}}{2 M}(\nabla \Phi) \cdot(\nabla \Phi)+\frac{i \hbar \sqrt{\rho}}{2 M} \nabla^{2} \Phi+V_{\operatorname{ext}} \sqrt{\rho}+U \rho \sqrt{\rho} \\
& -\mu_{\mathrm{c}} \sqrt{\rho}=0 .
\end{aligned}
$$

Separating the real and imaginary parts of Eq. (18), we find the following expression for the real part:

$$
\begin{gathered}
-\frac{\partial}{\partial t}\left(\frac{\Phi}{M}\right)+\frac{1}{2} \nabla\left(-\frac{\Phi}{M}\right) \cdot \nabla\left(-\frac{\Phi}{M}\right) \\
+\frac{U \rho}{M}+\frac{V_{\mathrm{ext}}}{M}+\frac{V_{\mathrm{Q}}}{M}-\frac{\mu_{\mathrm{c}}}{M}=0
\end{gathered}
$$

and for the imaginary part

$$
\frac{\partial \rho}{\partial t}+\nabla \cdot\left[\rho \nabla\left(-\frac{\Phi}{M}\right)\right]=0
$$

where

$$
V_{\mathrm{Q}} \equiv-\frac{\hbar^{2}}{2 M \sqrt{\rho}} \nabla^{2} \sqrt{\rho}
$$

is the so-called quantum potential. This quantum potential can be neglected when the Thomas-Fermi approximation is considered [36]. Note that, as it can be seen from Eq. (21), if the density is sufficiently small (and the term $\nabla^{2} \sqrt{\rho}$ is large enough), the quantum potential cannot be neglected [36].

Essentially, Eqs. (19) [more precisely, the gradient of Eq. (19)] and (20), under the Thomas-Fermi approximation, can be rewritten, respectively, as the Euler equation [Eq. (3)] and the continuity equation [Eq. (5)], where the flow velocity is $\vec{v}=-\nabla(\Phi / M)$, that implies that the BEC is irrotational.

Furthermore, comparing Eqs. (3) and (19), we obtain a relation between pressure and density, defining then an EoS for the BEC, namely

$$
P=\frac{U \rho^{2}}{2 M},
$$

which denotes that the BEC (in the Thomas-Fermi approximation) can be represented as an ideal fluid, which is barotropic and irrotational.

From Eq. (8) and the EoS (22), we may write the speed sound $c_{\mathrm{s}}$ in the $\mathrm{BEC}$ as

$$
c_{\mathrm{s}}=\sqrt{\frac{4 \pi a \hbar^{2} \rho}{M^{2}}} .
$$

To describe a BEC hydrodynamic vortex, we recall that the flow velocity has only an angular component, given by

$$
v_{\theta}=\frac{C}{r},
$$

with

$$
C \equiv \frac{\ell \hbar}{M},
$$

where $\ell$ is an integer number associated to the quantization of the circulation of the BEC hydrodynamic vortex $[35,36]$.

The density profile of the BEC may be determined directly from Eq. (19) [in the Thomas-Fermi approximation], being

$$
\rho=\frac{M}{4 \pi a \hbar^{2}}\left[\mu_{\mathrm{c}}-V_{\mathrm{ext}}-\frac{\hbar^{2} \ell^{2}}{2 M r^{2}}\right] .
$$

Before proceeding to the derivation of a physically acceptable expression for the density $\rho$ of the BEC hydrodynamic vortex, some considerations about the external potential $V_{\text {ext }}$ figuring in Eq. (26) are in order. In this paper, we consider a constant external potential, namely $V_{\text {ext }}=$ $V_{0}=$ constant $[35,36]$.

From Eq. (26), we may write an expression for the density profile of a vortex in a uniform medium, namely

$$
\rho=\rho_{\infty}\left(1-\frac{r_{\mathrm{c}}^{2}}{r^{2}}\right)
$$

with

$$
\rho_{\infty} \equiv \frac{M\left(\mu_{\mathrm{c}}-V_{0}\right)}{4 \pi a \hbar^{2}},
$$

where $\rho_{\infty}$ is density of the BEC at $r \rightarrow \infty$ (at large distances from the center of the vortex), and $r_{\mathrm{c}}$ is the so-called critical radius, given by

$$
r_{\mathrm{c}} \equiv \frac{|\ell|}{\sqrt{8 \pi a \rho_{\infty}}},
$$

defining the position where the density of the vortex goes to zero. The quantities $\rho_{\infty}$ and $a$ can be determined from the experimental setup [30]. Furthermore, from Eq. (28), the chemical potential $\mu_{\mathrm{c}}$ can be obtained, namely

$$
\mu_{\mathrm{c}}=\frac{4 \pi a \hbar^{2} \rho_{\infty}}{M}+V_{0} .
$$

Note that the critical radius $r_{\mathrm{c}}$ may be interpreted as a characteristic length that delimits where the Thomas-Fermi approximation is valid [36]; i.e., for sufficiently large distances from $r=r_{\mathrm{c}}$ [recalling that the density reaches its minimum value $(\rho \rightarrow 0)$ at $r \rightarrow r_{\mathrm{c}}$, the quantum potential $V_{\mathrm{Q}}$ is small enough and can be neglected [cf. Eq. (21)]. 
It is worth noting that the corresponding Kretschmann invariant [37] goes to infinity at the critical radius $r_{c}$, denoting that this point is an essential singularity $[15,28]$.

From Eqs. (23) and (27), we may write the speed of sound as

$$
c_{\mathrm{s}}=c_{s \infty} \sqrt{1-\frac{r_{\mathrm{c}}^{2}}{r^{2}}}
$$

where $c_{s \infty}=\sqrt{4 \pi a \hbar^{2} \rho_{\infty} / M^{2}}$ is the speed of sound at infinity. The speed of sound, as well as the density profile [cf. Eq. (27)], vanishes at the critical radius $r_{\mathrm{c}}$.

Using Eqs. (13), (24) and (31), we may obtain the outer boundary of the ergoregion, namely

$$
r_{\mathrm{e}}=\frac{\sqrt{3}|\ell|}{\sqrt{8 \pi a \rho_{\infty}}} .
$$

Comparing Eqs. (29) and (32), we find a relation between $r_{\mathrm{e}}$ and $r_{\mathrm{c}}$, as follows:

$$
\frac{r_{\mathrm{e}}}{r_{\mathrm{c}}}=\sqrt{3},
$$

denoting that the ergoregion encompasses the location where the density of the vortex vanishes: i.e., $r_{\mathrm{e}}>r_{\mathrm{c}}$.

\section{A. On the validity of the Thomas-Fermi approximation}

We have seen that under certain approximations, namely in the regime in which we can ignore the quantum potential, the background density is given by Eqs. (27)-(29) and furthermore, that the (phase) perturbations obey an effective Klein-Gordon equation in a curved spacetime with metric (11). In this section we will briefly analyze the conditions under which this effective formulation provides an accurate description of sound propagation in the vortex BEC.

As we have already discussed, the effective Klein-Gordon equation can be obtained by perturbing Eqs. (19) and (20), i.e., by expanding these equations to linear order in the phase pertubation $\phi=\delta \Phi$ and the density perturbaton $\varrho=\delta \rho$, solving the first equation for $\varrho$ in terms of $\phi$, and replacing the result in the second. In order to do so, we must ensure that the contribution from the quantum potential is negligible as compared with the interaction term. This amounts to the condition

$$
U \varrho \gg \frac{\hbar^{2}}{4 M \sqrt{\rho}}\left|\nabla^{2}\left(\frac{\varrho}{\sqrt{\rho}}\right)-\frac{\varrho}{\rho} \nabla^{2} \sqrt{\rho}\right| .
$$

From the second term we obtain the Thomas-Fermi approximation for the background density. This implies [using the lowest order expression (27)] that if we want it to be $\epsilon$ times smaller than the interaction term, we need that

$$
\frac{r-r_{\mathrm{c}}}{r_{\mathrm{c}}}>\frac{1}{\left(16 \ell^{2} \epsilon\right)^{1 / 3}}
$$

The first term gives the following upper bound for the wave number $k$ of the perturbations:

$$
k \ll \ell / r_{\mathrm{c}}=\sqrt{8 \pi a \rho_{\infty}} .
$$

Therefore, the effective Klein-Gordon description is only valid well beyond the central core of the vortex for low $\ell$, while for large $\ell$, the Thomas-Fermi approximation is valid at much closer distances. Even for low $\ell$, the analysis of the quasinormal modes for this type of vortices within the metric approximation is still interesting, among other things, because of the role played by the boundary conditions that have to be imposed close to the center.

\section{PERTURBATIONS IN A BEC HYDRODYNAMIC VORTEX}

Since the spacetime of the hydrodynamic vortex has a cylindrical symmetry, we may denote the angular dependence of the field $\phi$ as $e^{i m \theta}$ [cf. Eq. (7)], where $m$ is an integer number called the azimuthal number, which is related to the angular momentum of the perturbation. Furthermore, we may describe the propagation of the perturbation in the frequency domain, assuming that the field $\phi$ depends on time as $e^{i \omega t}$, with $\omega$ being the frequency of the perturbation. Then, the field $\phi$ can be written as follows:

$$
\phi(t, r, \theta, z)=\frac{1}{\sqrt{r}} \sum_{m=-\infty}^{\infty} u_{\omega m}(r) \exp [i(m \theta-\omega t)],
$$

where $u_{\omega m}(r)$ represent the single-frequency modes, i.e., the perturbations described in the frequency domain.

Using the covariant metric components $g^{\mu \nu}$, given by Eqs. (11), and (37) together with Eq. (6), we obtain the following ordinary differential equation:

$$
\begin{aligned}
& {\left[\rho \frac{d}{d r}\left(\rho \frac{d}{d r}\right)+\frac{\rho^{2}}{c_{\mathrm{s}}^{2}}\left(\omega-\frac{C m}{r^{2}}\right)^{2}\right.} \\
& \left.\quad-\frac{\rho^{2}}{r^{2}}\left(m^{2}-\frac{1}{4}\right)-\frac{\rho}{2 r} \frac{d \rho}{d r}\right] u_{\omega m}(r)=0 .
\end{aligned}
$$

We may substitute Eqs. (27) and (31) into Eq. (38), to obtain the following ordinary differential equation for a BEC hydrodynamic vortex:

$$
\begin{gathered}
{\left[x^{2}\left(3 x^{2}-1\right) \frac{d^{2}}{d x^{2}}+2 x \frac{d}{d x}+\left(\sqrt{3} \varpi x^{2}-\frac{\sqrt{2} m C}{|C|}\right)^{2}\right.} \\
\left.-\left(3 x^{2}-1\right)\left(m^{2}-\frac{1}{4}\right)-1\right] u_{\varpi m}(x)=0,
\end{gathered}
$$

where we defined a dimensionless frequency 


$$
\varpi \equiv \frac{\omega r_{\mathrm{e}}}{c_{\mathrm{s} \infty}},
$$

and a dimensionless radial coordinate

$$
x \equiv \frac{r}{r_{\mathrm{e}}} .
$$

Equation (39) has regular singular points at the origin and at the critical radius $x=x_{\mathrm{c}}\left(x_{\mathrm{c}} \equiv r_{\mathrm{c}} / r_{\mathrm{e}}=1 / \sqrt{3}\right)$, and an irregular singular point at spatial infinity.

\section{NUMERICAL METHODS}

In order to find the solutions of Eq. (39), we impose boundary conditions at a certain position $x=x_{\min }$ $\left(x_{\min }>x_{\mathrm{c}}\right)$ and at spatial infinity $x \rightarrow \infty$. We may assume a boundary condition of Neumann type at $x=x_{\min }$ (close to the center of the vortex) such that

$$
\left[\frac{d}{d x}\left(\frac{u_{\varpi m}(x)}{\sqrt{x}}\right)\right]_{x=x_{\min }}=0 .
$$

The boundary condition (42) can be physically interpreted as a cutoff, close to the center of the vortex, on the radial velocity increment $(\delta \vec{v}=-\nabla \phi)$ associated to the linear perturbation [cf. Eq. (37)]. This is related to a rigid barrier which has a great opposition to this increment of the velocity of the fluid (large acoustic impedance) placed at $r=r_{\min }$.

For large radial distances, in accordance with the asymptotic behavior of Eq. (39), we may write the following solution:

$$
u_{\varpi m}(x \rightarrow \infty) \sim \exp (i \varpi x) .
$$

We employed two numerical methods in the frequency domain to solve directly the ordinary differential equations (39), using the boundary conditions previously proposed, in order to obtain the frequency spectra $\varpi$ for different values of the azimuthal number $m$ and of $x_{\min }$.

\section{A. Direct integration method}

We may obtain the QNM frequencies from Eq. (39) determining their solutions via the direct integration (DI) method [12]. This procedure may be implemented as follows:

(i) We impose boundary conditions on the wave function $u_{\varpi m}(x)$ and its derivative at spatial infinity, namely

$$
u_{\varpi m}(x \rightarrow \infty)=\exp (i \varpi x) \sum_{j=0} \frac{b_{j}}{x^{j}},
$$

where $b_{j}$ are coefficients which can be determined by collecting the same inverse powers of $x$ from Eq. (39) at $x \rightarrow \infty$, keeping fixed the parameters $m$ and $\omega$. For example,

$$
b_{1}=\frac{i\left(-3+12 m^{2}+8 \sqrt{6} m \omega-4 \omega^{2}\right)}{24 \omega} .
$$

(ii) We integrate inwards Eq. (39), in the range $\infty>$ $x \geq x_{\min }$.

(iii) At $x=x_{\min }$, we extract the QNM frequencies as roots of $\left[\frac{d}{d x}\left(\frac{u_{\varpi m}(x)}{\sqrt{x}}\right)\right]_{x=x_{\min }}=0$, using a standard rootfinding algorithm such as Newton's method.

\section{B. Continued fraction method}

Another way to obtain the QNM frequencies from Eq. (39) consists in representing the wave function $u_{\varpi m}(x)$ as a Frobenius-like power series around $x=x_{\min }$ that satisfies the boundary conditions (42) and (43), as follows:

$$
u_{\varpi m}(x)=\exp (i \varpi x) \sum_{n=0} a_{n}\left(1-\frac{x_{\min }}{x}\right)^{n} .
$$

Substituting Eq. (45) into Eq. (39), we find the following five-term recurrence relation:

$\alpha_{0} a_{2}+\beta_{0} a_{1}+\gamma_{0} a_{0}=0$

$\alpha_{1} a_{3}+\beta_{1} a_{2}+\gamma_{1} a_{1}+\delta_{1} a_{0}=0$

$\alpha_{n} a_{n+2}+\beta_{n} a_{n+1}+\gamma_{n} a_{n}+\delta_{n} a_{n-1}+\epsilon_{n} a_{n-2}=0$,

for $n \geq 2$,

where the recurrence coefficients $\alpha_{n}, \beta_{n}, \gamma_{n}, \delta_{n}$ and $\epsilon_{n}$ are complex functions of the azimuthal number $m$ and $x_{\min }$, given by

$$
\begin{aligned}
\alpha_{n}= & -4 n(1+n)\left(-1+3 x_{\min }^{2}\right) \\
\beta_{n}= & 8 n\left[n\left(-2+3 x_{\min }^{2}\right)+i x_{\min }\left(1-3 x_{\min }^{2}\right) \omega\right] \\
\gamma_{n}= & 5-3 x_{\min }^{2}-12(-1+n) n\left(-2+x_{\min }^{2}\right) \\
& +12 m^{2}\left(-1+x_{\min }^{2}\right)-8 i[1+2(-1+n)] x_{\min } \omega \\
& +8 \sqrt{6} m x_{\min }^{2} \omega-4 x_{\min }^{2} \omega^{2} \\
\delta_{n}= & 6+24 m^{2}-16(-1+n)^{2}+8 i(-1+n) x_{\min } \omega \\
\epsilon_{n}= & -3-12 m^{2}-4(-1+n)+4(-1+n)^{2}
\end{aligned}
$$

being obtained from Eq. (39), together with Eqs. (45) and (46).

Using a double Gaussian elimination (cf. Refs. [14,38]), from the five-term recurrence relation (46) we may write the following three-term recurrence relation:

$$
\alpha_{n} a_{n+2}+\beta_{n} a_{n+1}+\gamma_{n} a_{n}=0, \quad \text { for } n \geq 0 .
$$

Using Eqs. (39), (45) and (47), it is possible to express analytically the recurrence coefficients $\alpha_{n}, \beta_{n}$, and $\gamma_{n}$, as functions of the parameters $m, \omega$ and $x_{\min }$.

Considering the boundary condition (42), Eqs. (45) and (47), we may obtain the following continued-fraction (CF) [39]:

$$
1-2 i \varpi x_{\min }+\frac{2 \gamma_{1}}{\beta_{1}-\frac{\alpha_{1} \gamma_{2}}{\beta_{2}-\frac{\alpha_{2} \gamma_{3}}{\beta_{3}-\cdots}}}=0
$$



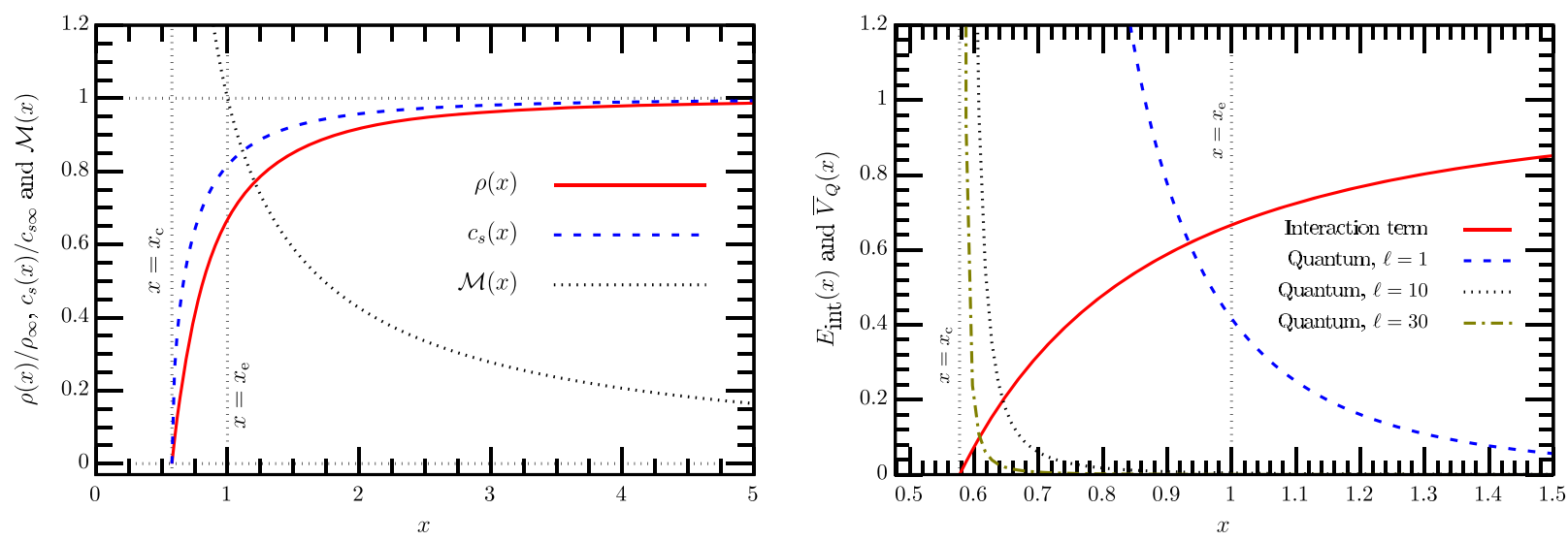

FIG. 1. Left: Fluid density $\rho$, speed of sound $c_{\mathrm{s}}$, and local Mach number $\mathcal{M}$, for the BEC hydrodynamic vortex, as functions of $x$. We also exhibit the position of the critical radius, $x_{\mathrm{c}} \equiv 1 / \sqrt{3}$, and of the outer boundary of the ergoregion, $x_{\mathrm{e}} \equiv 1$ (vertical dashed lines). Right: Dimensionless interaction term, $E_{\text {int }}(x)$ given by Eq. (49), and dimensionless quantum potential, $\bar{V}_{Q}(x)$ given by Eq. (50), for $\ell=1,10$ and 30 .

Again, we may obtain the QNM frequencies from Eq. (48) for different values of the azimuthal number $m$ and $x_{\min }$, using a standard root-finding algorithm such as Newton's method.

\section{ERGOREGION INSTABILITY}

In this section we investigate the ergoregion instability of the BEC hydrodynamic vortex.

In the left frame of Fig. 1 we plot the fluid density $\rho$ [given by Eq. (27)], the speed of sound $c_{\mathrm{s}}$ [given by Eq. (31)] and the local Mach number $\mathcal{M} \equiv|\vec{v}| / c_{\mathrm{s}}$, as functions of $x$. In the right frame of Fig. 1 we plot the dimensionless interaction term given by

$$
E_{\text {int }}(x) \equiv \frac{U \rho(x)}{K_{\mathrm{c}}},
$$

and the dimensionless quantum potential

$$
\bar{V}_{Q}(x) \equiv \frac{V_{Q}(x)}{K_{\mathrm{c}}},
$$

with $K_{\mathrm{c}} \equiv 4 \pi a \hbar^{2} \rho_{\infty} / M$. We note that as the density of the BEC decreases to zero at $x \rightarrow x_{\mathrm{c}}$, the quantum potential can assume a significant value [cf. Eq. (21)], being non negligible, and the Thomas-Fermi approximation is no longer valid in this case. Furthermore, we note that the larger the value of $\ell$ is, the closer to the vortex the dimensionless quantum potential goes to zero. In our numerical simulations, we consider boundary conditions imposed at sufficiently large distances for the critical radius, e.g., for a range of values of $x_{\min }$ with $x_{\min } \geq 0.7$ (with $x_{\min } / x_{\mathrm{c}}>1.2$ ).

Using two frequency domain methods (DI and CF methods), we computed the QNM frequencies. In order to verify the stability of the modes, boundary conditions of
Neumann type are applied, outside and inside of the ergoregion, for this compressible acoustic system.

For the QNM analysis, we assume the standard convention of ordering the imaginary part of the QNM frequencies $\varpi$ [25]. The fundamental mode $(n=0)$ is the one with the largest imaginary part of the QNM frequencies. Thus, if the mode is unstable $[\operatorname{Im}(\varpi)>0]$, the fundamental mode corresponds to the smallest instability time scale, and for stable modes $[\operatorname{Im}(\varpi)<0]$, it corresponds to the longestlived mode.

TABLE I. QNM frequencies $\varpi$ of the BEC hydrodynamic vortex for different values of the azimuthal number $m$, for $x_{\min }=2.0$ (outside the ergoregion) and $x_{\min }=0.7$ (inside the ergoregion), obtained numerically from estimates via DI and CF methods [41]. At $x=x_{\min }$, we imposed the boundary condition (42), and, at spatial infinity, we considered the boundary condition (43).

\begin{tabular}{lccc}
\hline \hline \multicolumn{4}{c}{$x_{\min }=2.0$ (outside the ergoregion) } \\
\hline$m$ & Method & $\operatorname{Re}(\varpi)$ & $\operatorname{Im}(\varpi)$ \\
\hline 5 & DI & -1.315457 & -0.240587 \\
& CF & -1.315457 & -0.240587 \\
6 & DI & -1.590645 & -0.246052 \\
& CF & -1.590645 & -0.246052 \\
7 & DI & -1.865445 & -0.250641 \\
& CF & -1.865445 & -0.250641 \\
8 & DI & -2.139994 & -0.254608 \\
& CF & -2.139994 & -0.254608 \\
\hline \hline \multicolumn{4}{c}{} \\
\hline$m$ & $x_{\min }=0.7$ (inside the ergoregion) \\
\hline 5 & Method & $\operatorname{Re}(\varpi)$ & $\operatorname{Im}(\varpi)$ \\
6 & DI & +1.375559 & $+9.448427 \times 10^{-7}$ \\
7 & DI & +1.985322 & $+2.932041 \times 10^{-7}$ \\
8 & DI & +2.625150 & $+8.056820 \times 10^{-8}$ \\
\hline \hline
\end{tabular}




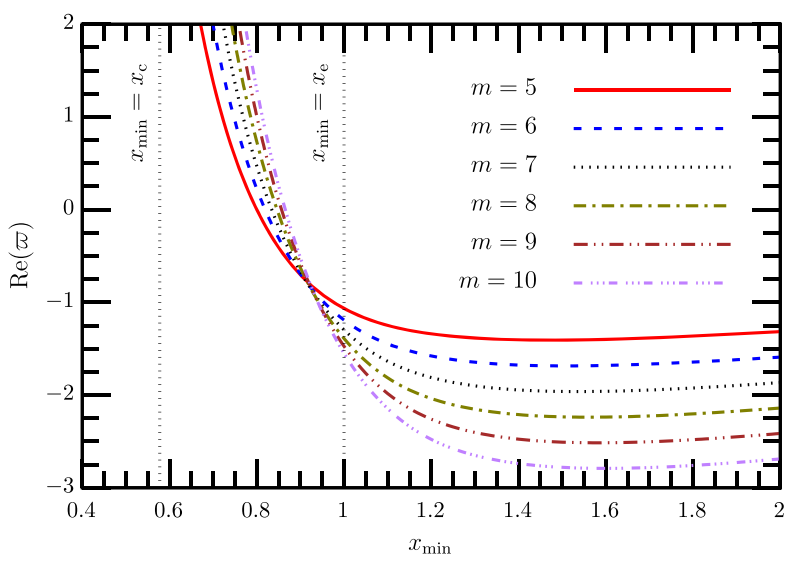

FIG. 2. Real part of the fundamental $(n=0)$ QNM frequencies $\varpi$ of the BEC hydrodynamic vortex, for azimuthal numbers $m=5,6,7,8,9$ and 10 , as a function of $x_{\min }$, obtained via DI and CF methods [41]. At $x=x_{\min }$, we imposed a boundary condition of the Neumann type, given by Eq. (42), and at spatial infinity we considered the asymptotic behavior given by Eq. (43).

From Eq. (39), it can be clearly seen that there are symmetries associated to the frequency $\varpi$, relating the corotating modes $(\mathrm{Cm}>0)$ and the counterrotating ones $(\mathrm{Cm}<0)$, as follows:

$$
\varpi(C m>0)=-\varpi^{*}(C m<0),
$$

where "*" denotes complex conjugation. From Eq. (51), we note that to each QNM frequency of a corotating mode there is a corresponding one of a counterrotating mode with opposite real part and the same imaginary part [40]. Henceforth, taking into account the symmetries (51), we may assume, without loss of generality, that $m>0$ and $C>0$.
Estimates of the QNM frequencies $\varpi$ are exhibited in Table I, considering different values of the azimuthal number $m$ and applying the boundary condition (42) at two different positions of the radial coordinate. These QNM frequencies are obtained via DI and CF methods [41]. Note that, as the azimuthal number $m$ is increased, the magnitude of the real and imaginary parts of the QNM frequencies for the stable modes also increases, while the opposite happens for the unstable modes. The ergoregion instability can be more easily perceived for large values of $m$.

In Figs. 2 and 3 we plot, respectively, the real and imaginary parts of the fundamental $(n=0)$ QNM frequencies $\varpi$, for azimuthal numbers $m=5,6,7,8,9$ and 10, obtained via DI and CF methods [41]. We observe a decrease of the magnitude of the real and imaginary parts of the QNM frequencies for stable modes and an increase for the unstable modes. From Fig. 2, we may observe that there exists a certain point (around $x_{\min } \approx 0.9$ ), in which the real part of the QNM frequencies is the same for all azimuthal numbers $m$. We should point out that this pattern of coincidence does not happen if we, instead of the boundary condition of Neumann type, choose boundary condition of Dirichlet type. We also note that, as we can clearly see in the right plots of Fig. 3, the threshold between stability and instability is smaller for BEC hydrodynamic vortex than for the polytropic hydrodynamic vortex (with the setup described in Ref. [15]); i.e., the transition from stability to instability occurs more rapidly for the BEC hydrodynamic vortex than for the polytropic hydrodynamic vortex with a compatible experimental setup in a perfect fluid (note that the transition of stability to instability is more sudden for azimuthal number $m=5$ than for $m \geq 6$ ).
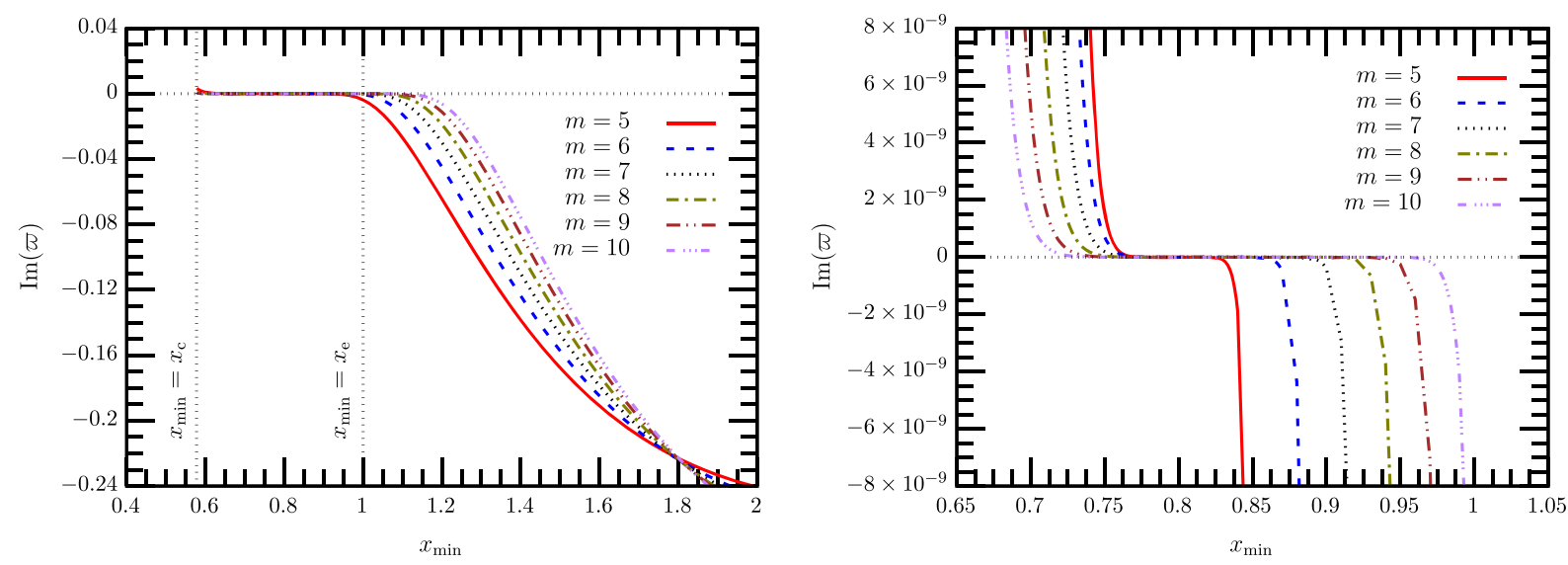

FIG. 3. Imaginary part of the fundamental $(n=0)$ QNM frequencies $\varpi$ of the BEC hydrodynamic vortex (left frame, with a zoom in the right frame), for azimuthal numbers $m=5,6,7,8,9$ and 10, as a function of $x_{\min }$, obtained via DI and CF methods [41]. At $x=x_{\min }$, we imposed a boundary condition of the Neumann type, given by Eq. (42), and at spatial infinity we considered the asymptotic behavior given by Eq. (43). 


\section{CONCLUSION}

We investigated the ergoregion instability of a purely circulating system of ideal fluid (representing a quantum system): the BEC hydrodynamic vortex. We have shown that, by imposing boundary conditions inside the ergoregion of this purely circulating system, instabilities appear, which are associated with the existence of an ergoregion (supersonic flow regime) and absence of an event horizon [14-16]. From the QNM analysis, we concluded that the imaginary part of the QNM frequencies of this system is positive when the boundary conditions are imposed sufficiently inside the ergoregion. Furthermore, we have shown that, as the position of the boundary condition is placed more inside of the ergorigion, the system is more unstable, with the transition from stability to instability being more sudden for the BEC hydrodynamic vortex than for the polytropic hydrodynamic vortex with a compatible experimental setup in a perfect fluid [15]. We have thus verified for a quantum system a relevant property associated with effective spacetime with ergoregion and without an event horizon, namely the ergoregion instability.

\section{ACKNOWLEDGMENTS}

We thank Vitor Cardoso for the scientific collaboration that motivated the present study. The authors would like to thank Conselho Nacional de Desenvolvimento Científico e Tecnológico (CNPq), and Coordenação de Aperfeiçoamento de Pessoal de Nível Superior (CAPES), in Brazil, for partial financial support. Financial support was also provided by the Spanish MINECO through the Projects No. FIS2014-54800C2-2-P and No. FIS2017-86497-C2-2-P (with FEDER contribution).
[1] W. G. Unruh, Experimental Black Hole Evaporation, Phys. Rev. Lett. 46, 1351 (1981).

[2] M. Visser, Acoustic black holes: Horizons, ergospheres, and Hawking radiation, Classical Quantum Gravity 15, 1767 (1998).

[3] Analogue Spacetimes: The First Thirty Years, edited by V. Cardoso, L. C. B. Crispino, S. Liberati, E. S. Oliveira, and M. Visser (Editora Livraria da Física, São Paulo, 2013).

[4] C. Barcelo, S. Liberati, and M. Visser, Analogue gravity, Living Rev. Relativity 8, 12 (2005); Analogue gravity 14, 3 (2011).

[5] M. Visser, C. Barcelo, and S. Liberati, Analog models of and for gravity, Gen. Relativ. Gravit. 34, 1719 (2002).

[6] L. C. B. Crispino, E. S. Oliveira, and G. E. A. Matsas, Absorption cross section of canonical acoustic holes, Phys. Rev. D 76, 107502 (2007).

[7] E. S. Oliveira, S. R. Dolan, and L. C. B. Crispino, Absorption of planar waves in a draining bathtub, Phys. Rev. D 81, 124013 (2010).

[8] S. R. Dolan, E. S. Oliveira, and L. C. B. Crispino, Scattering of sound waves by a canonical acoustic hole, Phys. Rev. D 79, 064014 (2009).

[9] S. R. Dolan, E. S. Oliveira, and L. C. B. Crispino, AharonovBohm effect in a draining bathtub vortex, Phys. Lett. B 701, 485 (2011).

[10] E. Berti, V. Cardoso, and J. P. S. Lemos, Quasinormal modes and classical wave propagation in analogue black holes, Phys. Rev. D 70, 124006 (2004).

[11] V. Cardoso, J. P. S. Lemos, and S. Yoshida, Quasinormal modes and stability of the rotating acoustic black hole: Numerical analysis, Phys. Rev. D 70, 124032 (2004).

[12] S. R. Dolan, L. A. Oliveira, and L. C. B. Crispino, Quasinormal modes and Regge poles of the canonical acoustic hole, Phys. Rev. D 82, 084037 (2010).
[13] S. R. Dolan, L. A. Oliveira, and L. C. B. Crispino, Resonances of a rotating black hole analogue, Phys. Rev. D 85, 044031 (2012).

[14] L. A. Oliveira, V. Cardoso, and L. C. B. Crispino, Ergoregion instability: The hydrodynamic vortex, Phys. Rev. D 89, 124008 (2014).

[15] L. A. Oliveira, V. Cardoso, and L. C. B. Crispino, Quasinormal modes of the polytropic hydrodynamic vortex, Phys. Rev. D 92, 024033 (2015).

[16] L. A. Oliveira, V. Cardoso, and L. C. B. Crispino, Superresonant instability of a compressible hydrodynamic vortex, Int. J. Mod. Phys. D 25, 1641019 (2016).

[17] L. A. Oliveira and L. C. B. Crispino, Isothermal perfect fluid as a hydrodynamic vortex: Quasinormal modes investigation, Int. J. Mod. Phys. D 27, 1843013 (2018).

[18] S. Hod, Onset of superradiant instabilities in the hydrodynamic vortex model, Phys. Rev. D 90, 027501 (2014).

[19] C. L. Benone, L. C. B. Crispino, C. Herdeiro, and E. Radu, Acoustic clouds: Standing sound waves around a black hole analogue, Phys. Rev. D 91, 104038 (2015).

[20] L. J. Garay, J. R. Anglin, J. I. Cirac, and P. Zoller, Black Holes in Bose-Einstein Condensates, Phys. Rev. Lett. 85, 4643 (2000).

[21] L. J. Garay, J. R. Anglin, J. I. Cirac, and P. Zoller, Sonic black holes in dilute Bose-Einstein condensates, Phys. Rev. A 63, 023611 (2001).

[22] C. Barcelo, S. Liberati, and M. Visser, Probing semiclassical analog gravity in Bose-Einstein condensates with widely tunable interactions, Phys. Rev. A 68, 053613 (2003).

[23] J. Steinhauer, Observation of self-amplifying Hawking radiation in an analog black hole laser, Nat. Phys. 10, 864 (2014).

[24] J. Steinhauer, Measuring the entanglement of analogue Hawking radiation by the density-density correlation function, Phys. Rev. D 92, 024043 (2015). 
[25] E. Berti, V. Cardoso, and A. O. Starinets, Quasinormal modes of black holes and black branes, Classical Quantum Gravity 26, 163001 (2009).

[26] K. D. Kokkotas and B. G. Schmidt, Quasinormal modes of stars and black holes, Living Rev. Relativity 2, 2 (1999).

[27] H. P. Nollert, Quasinormal modes: The characteristic "sound" of black holes and neutron stars, Classical Quantum Gravity 16, R159 (1999).

[28] C. Cherubini and S. Filippi, Acoustic metric of the compressible draining bathtub, Phys. Rev. D 84, 084027 (2011).

[29] C. Barcelo, A. Cano, L. J. Garay, and G. Jannes, Quasinormal mode analysis in BEC acoustic black holes, Phys. Rev. D 75, 084024 (2007).

[30] T. R. Slatyer and C. M. Savage, Superradiant scattering from a hydrodynamic vortex, Classical Quantum Gravity 22, 3833 (2005).

[31] M. Visser and S. E. C. Weinfurtner, Vortex geometry for the equatorial slice of the Kerr black hole, Classical Quantum Gravity 22, 2493 (2005).

[32] U. R. Fischer and M. Visser, Riemannian Geometry of Irrotational Vortex Acoustics, Phys. Rev. Lett. 88, 110201 (2002).

[33] P. Marecki and R. Schützhold, Whispering gallery like modes along pinned vortices, JETP Lett. 96, 674 (2013).

[34] P. Marecki, Propagation of sound on line vortices is superfluids: Role of ergoregions, J. Phys. A 45, 295501 (2012).
[35] C. J. Pethick and H. Smith, Bose-Einstein Condensation in Dilute Gases (Cambridge University Press, Cambridge, 2002).

[36] L. Pitaesvskii and S. Stringary, Bose-Einstein Condensation and Superfluidity (Oxford University Press, New York, 2016).

[37] R. Wald, General Relativity (The University of Chicago Press, Chicago, 1984).

[38] H. Onozawa, T. Mishima, T. Okamura, and H. Ishihara, Quasinormal modes of maximally charged black holes, Phys. Rev. D 53, 7033 (1996).

[39] E. W. Leaver, An Analytic representation for the quasi normal modes of Kerr black holes, Proc. R. Soc. A 402, 285 (1985).

[40] Interestingly, the symmetries of the spectrum, denoted in Eq. (51), are quite different when compared to the ones of the draining bathtub case (cf. Ref. [13]). Essentially, this difference of symmetries for these two systems is related to the ingoing boundary condition applied in each case. For the draining bathtub case, the ingoing boundary condition explicitly depends on the product $\mathrm{Cm}$ [cf. Eq. (12) in Ref. [13]], differently from the hydrodynamic vortex case [cf. Eq. (42)].

[41] We used only the DI method to compute QNM frequencies $\varpi$ for $x_{\min }<1$, since that CF method does not converge sufficiently well in this range. 